\title{
RESEARCH
}

Open Access

\section{Porcine epidemic diarrhea virus S1 protein is the critical inducer of apoptosis}

Yifeng Chen ${ }^{1,2}$, Zhibang Zhang ${ }^{1}$, Jie Li ${ }^{1}$, Yueyi Gao', Lei Zhou' ${ }^{1}$ Xinna Ge${ }^{1}$, Jun Han ${ }^{1}$, Xin Guo ${ }^{1 *}$ (D and Hanchun Yang ${ }^{1}$

\begin{abstract}
Background: Porcine Epidemic Diarrhea (PED) is an acute and highly contagious enteric disease caused by PED virus (PEDV), characterized by vomitting, watery diarrhea and fatal dehydration with high mortality in sucking piglets of one week of age. Although PEDV induced cell apoptosis has been established in vitro and in vivo, the functional protein that contributes to this event remains unclear.
\end{abstract}

Methods: The activation or cleavage of main apoptosis-associated molecular such as AIFM1, caspase-3, caspase-8, caspase-9 and PARP in PEDV infected host cells were analyzed by western blotting. The nuclear change of infected cell was monitored by confocal immunofluorescence assay. The overexpressing plasmids of 16 non-structural proteins (Nsp1-16) and 6 structural proteins (M, N, E, ORF3, S1 and S2) were constructed by cloning. Cell apoptosis induced by PEDV or overexpression non-structural or structural proteins was measured by the flow cytometry assay.

Results: PEDV could infect various host cells including Vero, Vero-E6 and Marc-145 and cause obvious cytopathic effects, including roundup, cell fusion, cell membrane vacuolation, syncytium formation and cause apparent apoptosis. In infected cells, PEDV-induced apoptosis is accompanied by nuclear concentration and fragmentation as a result of caspase-3 and caspase-8 activation and AIFM1 and PARP cleavage. Overexpression of S1 Spike protein of PEDV SM98 strain effectively induced host cell apoptosis, while the expression of the other non-structure proteins (Nsp1-16) and structural proteins (M, N, E, S2 and ORF3) has no or less effect on cell apoptosis. Similarly, expression of S1 protein from wild-type strain BJ2011 or cell-adapted strain CV777, also induce apoptosis in transfected cells. Finally, we demonstrated that the S1 proteins from various coronavirus family members such as TGEV, IBV, CCOV, SARS and MERS could also induce Vero-E6 cells apoptosis.

Conclusion: S1 Spike protein is one of the most critical functional proteins that contribute to cell apoptosis. Expression of S1 proteins of the coronavirus tested in this study could all induce cell apoptosis suggesting S1 maybe is an effective inducer in Coronavirus-induced cell apoptosis and targeting S1 protein expression probably is a promising strategy to inhibit coronavirus infection and thus mediated apoptosis on host cells.

Keywords: Porcine epidemic diarrhea virus (PEDV), Spike S1 protein, Apoptosis, Apoptosis-inducing factor mitochondria associated 1 (AIFM1)

\footnotetext{
* Correspondence: guoxincau@cau.edu.cn

${ }^{1}$ Key Laboratory of Animal Epidemiology of the Ministry of Agriculture,

College of Veterinary Medicine and State Key Laboratory of

Agrobiotechnology, China Agricultural University, No.2 Yuanmingyuan West

Road, Haidian Distract, Beijing 100193, People's Republic of China

Full list of author information is available at the end of the article
}

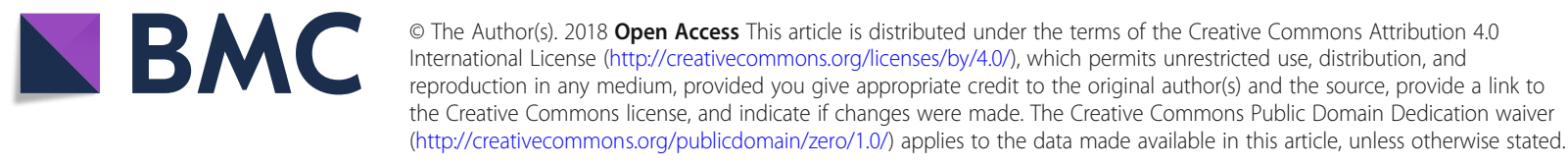




\section{Background}

Porcine Epidemic Diarrhea (PED) is an acute and highly contagious enteric disease characterized by severe watery diarrhea, dehydration, and anorexia. Deceased piglets presented with thin and almost transparent small intestines containing undigested milk curdles. The etiological agent PED virus (PEDV) was first isolated and recognized from Europe in the 1970s [1, 2], then it was spread and prevalent in Asian for decades [3, 4]. PEDV originally caused a relatively mild and sporadic disease. However, since more virulent variant strains appeared in 2010 [5-8], PEDV has been subsequently associated with severe outbreaks of diarrheal disease [9] in Asia and in North American [10-13]. Acute PEDV outbreaks normally resulted in enormous economic losses to swine industries around the world, for instance, in 2013 to 2014 PEDV killed more than 7 million pigs in the North American [14]. Currently, PEDV poses a serious threat to the swine industry worldwide.

PEDV is an enveloped single-stranded and positivesense RNA virus, belongs to the genus Alpha coronavirus, family Coronaviridae, order Nidovirales [2]. The genome of PEDV is about $28 \mathrm{~kb}$ and comprises of a $5^{\prime}$ untranslated region (UTR), at least 7 open reading frames (ORF1a, ORF1b, and ORF2-6), and a 3' UTR. The ORF $1 \mathrm{a}$ and $1 \mathrm{~b}$ cover the $5^{\prime}$-proximal two-thirds of the genome coding for replicase polyprotein (pp) la and pplab, respectively $[15,16]$. These ppla and pplab can be cleaved by internal proteases generating 16 nonstructural proteins, namely nsp1-16. ORF2-6 encode four structural proteins including the spike $(\mathrm{S})$, envelope $(\mathrm{E})$, membrane $(\mathrm{M})$ and nucleocapsid $(\mathrm{N})$ proteins, while ORF3 encodes an accessory protein [15]. The large spikes on the coronavirus envelope are composed of trimers of the spike proteins. The spike protein mediates viral entry into host cells by functioning as a class I viral fusion protein [17]. During maturation, the spike protein is often cleaved into a receptor-binding subunit S1 and a membrane-fusion subunit S2 [18].

PEDV induce infected host cell apoptosis has been established in vitro and in vivo [19]. PEDV also induces caspase-independent apoptosis in host cells by the activation of mitochondrial apoptosis-inducing factor based on the observation of AIFM1 relocated into the nucleus during the PEDV infection [19]. However, the protein that is mainly responsible for this induction remains unclear. In this study, we have provided evidence that $\mathrm{S} 1$ protein is the main inducer for cell apoptosis during PEDV infection.

\section{Methods \\ Cells and viruses}

Vero, Vero-E6, MARC-145 and BHK-21 cells were obtained from American Type Culture Collection (ATCC), all the cells were maintained in Dulbecco Modified Eagle Medium (DMEM; Invitrogen) supplemented with 10\% fetal bovine serum (FBS) and antibiotics $(100 \mathrm{U} / \mathrm{ml}$ of

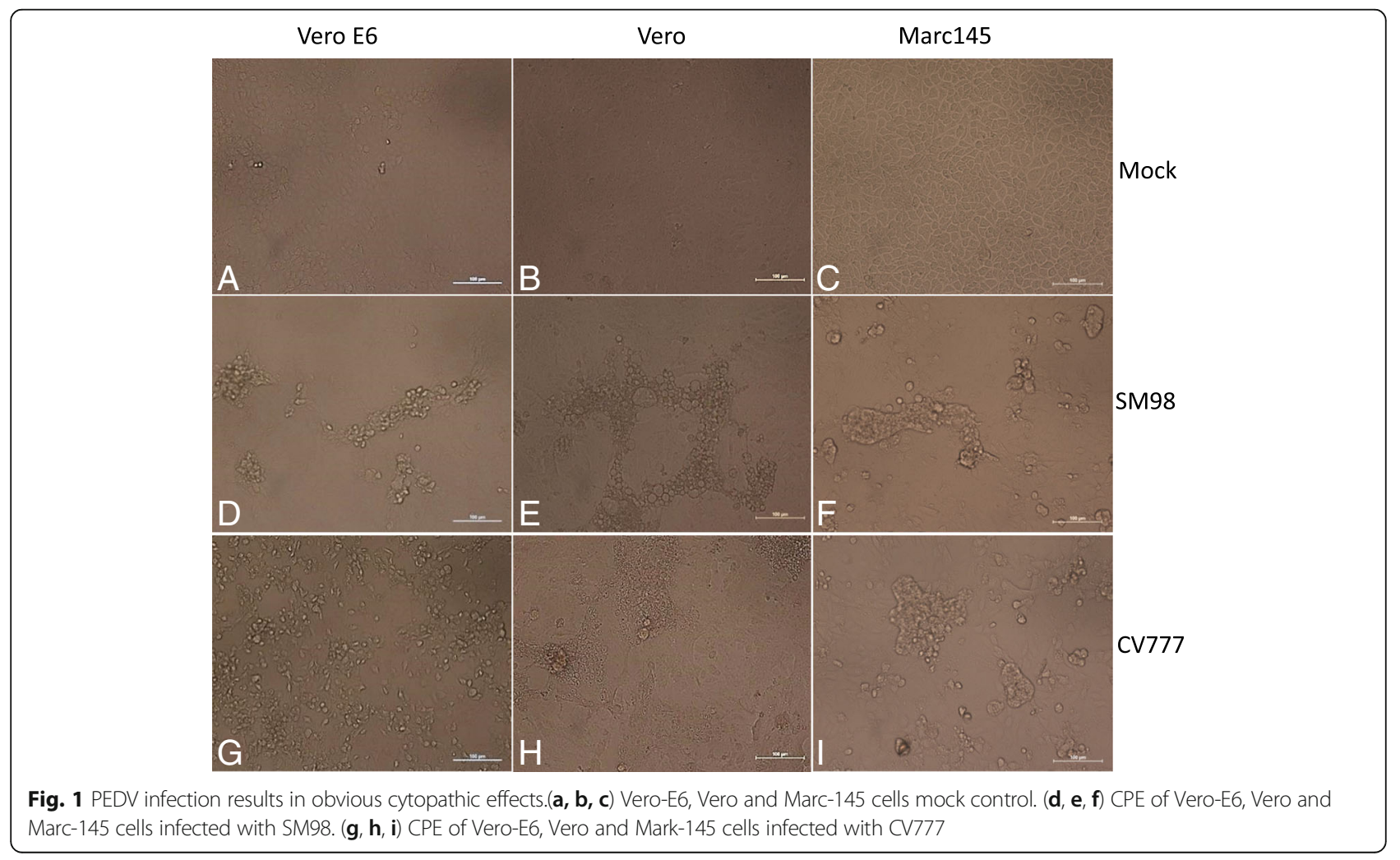


penicillin and $100 \mu \mathrm{g} / \mathrm{ml}$ of streptomycin) in a $5 \% \mathrm{CO}_{2}$ incubator. PEDV SM98 and CV777 strains were kept in our laboratory, wild-type strains BJ2011 was isolated from a farm in Beijing in 2011.

\section{Reagents}

Fluorescein (FITC)-conjugated affiniPure Goat anti-Mouse IgG $(\mathrm{H}+\mathrm{L})$ was a product of Jackson Immuno Research; Anti-PEDV Spike mAb, Annexin V-PE/7-AAD Apoptosis Detection Kit was purchased from BD; Caspase-3, caspase8, caspase-9 and PARP rabbit mAbs were bought from Cell Signaling Technology; Anti-AIFM1 mice mAb was a product of Sigma-Aldrich; Caspase-3, -8 and -9 activity assay kits were purchased from Biovision.

\section{Plasmids construction}

The Nsp1-16, M, N, E, ORF3, S1 and S2 genes were cloned from PEDV strain SM98 using specific primers (Additional file 1: Table S1). Primers were synthesized by BGI Tech Solutions Co., Ltd. (Beijing, China). All the plasmids were constructed by standard recombined DNA techniques. Briefly, all the non-structural and structural genes were amplified by RT-PCR from PEDV RNA genome and cloned into the pEGFP-N1 vector. The $\mathrm{S} 1$ genes of transmissible gastroenteritis virus (TGEV), avian infectious bronchitis virus (IBV) and canine coronavirus virus $(\mathrm{CCoV})$ were cloned from vaccine strains (TGEV $\mathrm{H}$ Strain, IBV-H52 strain and CCoV K378 strain, respectively). The S1 genes of Middle East respiratory syndrome (MERS) coronavirus and Severe acute respiratory syndrome (SARS) coronavirus were synthesized by Genscript according to the sequences submitted in Genebank (accession number: KY581693.1 and AH013709.2, respectively).

\section{Apoptosis assay}

Vero, Vero-E6 and Marc-145 cells were seeded in sixwell-plate and cultured overnight, cells were then mock-

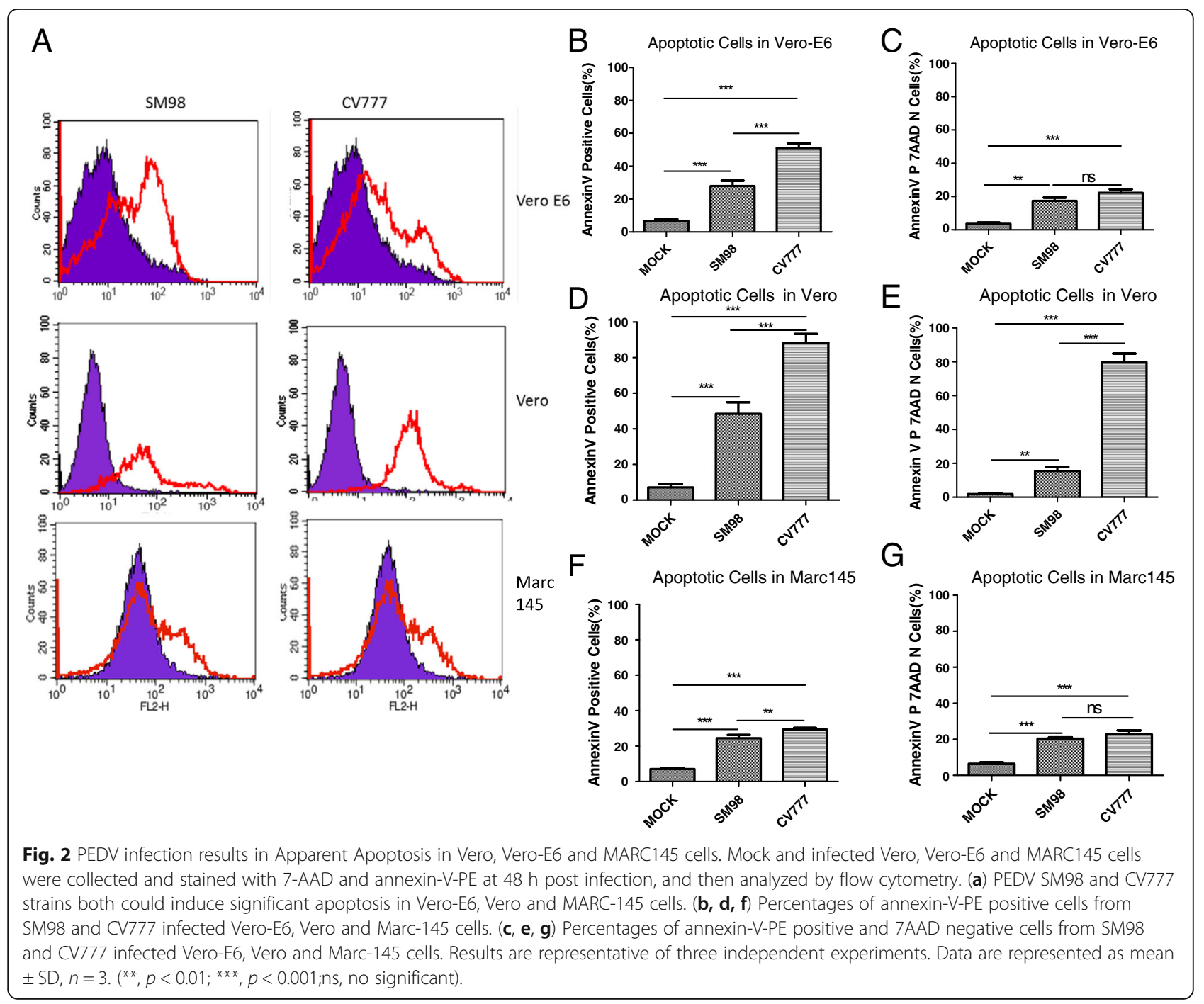


infected or infected with PEDV CV777 or SM98 strains at a multiplicity of infection (MOI) of 0.1 for $48 \mathrm{~h}$. Cells were trypsinized and resuspended with buffer $(135 \mathrm{mM}$ $\mathrm{NaCl}, 10 \mathrm{mM}$ HEPES, $5 \mathrm{mM} \mathrm{CaCl}$ ) and stained with Annexin V-PE and 7-AAD at room temperature for $15 \mathrm{~min}$. Cells were analyzed by flow cytometry. Fluorescence-activated cells sorter (FACS) data were analyzed using CellQuest software (BD).

Vero-E6 or BHK21 cells were cultured in six-well-plate and grow to $70-80 \%$ confluent, cells were transfected with various plasmids including pEGFP-N1, pEGFP-Nsp1-16, pEGFP-M, pEGFP-N, pEGFP-E, pEGFP-ORF3, pEGFP-S1 and pEGFP-S2 by Lipofectamine LTX. After $48 \mathrm{~h}$ transfection, cells were harvested and stained with Annexin V-PE and 7-AAD at room temperature for $15 \mathrm{~min}$. GFP-positive cells were gated for apoptosis analysis. FACS data were analyzed with CellQuest software (BD).

\section{Western blotting}

Protein samples were separated by SDS-PAGE and were electrically transferred onto a polyvinylidene fluoride (PVDF) membrane. After being blocked with 5\% skim milk in phosphate-buffered saline (PBS), the membrane was incubated with proper antibodies and subsequently probed with appropriated horseradish peroxidase (HRP) conjugated goat anti-mouse secondary antibody. The protein bands were developed with the ECL western blotting system (Fisher Scientific) and exposed to a fluorchem E apparatus (Proteinsimple, Santa Clara, CA,
USA). Western blotting bands are quantified according to intensity by using ImageJ software, normalized to $\beta$-actin and expressed relative to mock infection.

\section{Confocal microscopy assays}

Vero cells were grown on coverslips in 24-well-plate (Costar, Corning Incorporation) and infected with the SM98 virus at an MOI of 0.1, uninfected cells serve as a mock control. The cells were fixed and permeabilized with cold anhydrous ethanol for $20 \mathrm{~min}$ at room temperature (RT) at 24, 48 and $72 \mathrm{~h}$ post infection, respectively. Followed by being blocked with $2 \%$ BSA in PBS for $1 \mathrm{~h}$ at RT. Then, the cells were incubated with mice anti-PEDV $\mathrm{S}$ monoclonal antibody at $4{ }^{\circ} \mathrm{C}$ in a humid chamber. After being rinsed 3 times with PBS, the cells were incubated with fluorescein isothiocyanate (FITC)-conjugated goat anti-mice antibody for $1 \mathrm{~h}$ at RT. Finally, the cells were stained with DAPI, and the images were viewed under an Olympus confocal microscope (fluoview 1000×).

\section{Caspase- $3,-8$ and -9 activity assays}

Vero cells were seeded on six-well-plate and were mockinfected or infected with SM98 strain at an MOI of 1. Infected cells were incubated for $48 \mathrm{~h}$ before trypsinized. Cells were washed with cold PBS, resuspended in $50 \mu \mathrm{L}$ of chilled cell lysis buffer, and incubated on ice for $15 \mathrm{~min}$. The cell lysates were centrifuged at 10,000×g for $15 \mathrm{~min}$. The supernatants were collected and frozen at $-70{ }^{\circ} \mathrm{C}$,

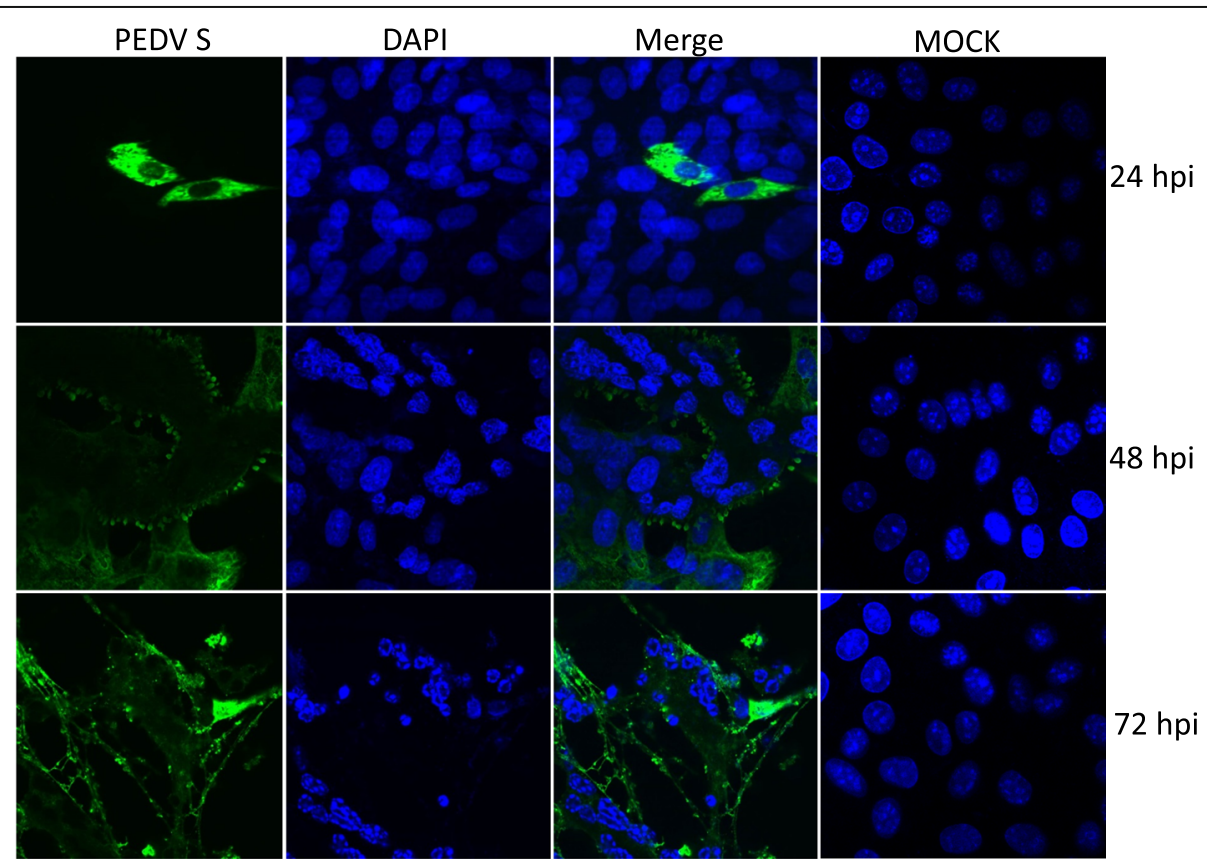

Fig. 3 PEDV infection results in cell nuclear DAN fragmentation and chromatin condensation. Vero cells grown on coverslips were infected with PEDV SM98 at a multiplicity of infection of 0.1, The cells were fixed and incubated with mice anti PEDV S monoclonal antibody and DAPI at 12 , 48 and $72 \mathrm{~h}$ post infection. (fluoview 1000x) 
the activities of caspase- $3,-8$ and -9 in samples were measured by colorimetric assay with a plate reader.

\section{Statistical analysis}

The statistically significant differences between PEDV infected cells and controls in the induction of apoptosis and caspase activation and, between the recombinants plasmid transfected cells and pEGFP-N1 transfected control in the induction of apoptosis was determined by unpaired T-tests or ANOVA accordingly with GraphPad Prism (Version 5.0) software. Differences were considered statistically at a value of $p<0.05$.

\section{Results}

Cytopathogenic effect produced by PEDV strains SM98 and CV777 infection

When PEDV SM98 and CV777 strains were inoculated into Vero, vero-E6 and Mark-145 cells respectively, distinct cytopathic effects (CPEs) including roundup (Fig. 1 $\mathrm{d}$ and g), cell fusion (F and I), vacuolation (E), syncytium $(\mathrm{H})$ and detachment were observed, as shown in Fig. 1.
PEDV infection induces apoptosis in multiple host cells Mock and infected Cells were collected at $48 \mathrm{~h}$ post-infection and stained by Annexin V-PE/7-AAD and analyzed by flow cytometry. As shown in Fig. 2a, SM98 and CV777 strains infection induce significant apoptosis in Vero, Vero-E6 and Marc-145 cells, no matter total apoptosis or early apoptosis. Percentages of Annexin-V-PE positive cells from SM98 and CV777 infected Vero-E6, Vero and Marc-145 cells are shown in Fig. 2b, $d$ and $f$ respectively, while percentages of Annexin-V-PE positive and 7AAD negative cells are shown in Fig. 2c, e and g.

\section{PEDV infection resulted in syncytium formation and cell} nuclear DAN fragmentation and chromatin condensation The nuclear change of PEDV infected cells was monitored by confocal immunofluorescence assay. As can be seen in Fig. 3, with the PEDV replication, the nuclear of infected cells gradually gathered, piled up, some of which split into fragments. These gathered nuclears and nuclear fragments and the surrounded cytoplasmic membrane formed a multinucleated syncytium. At the end stage of infection (72 $\mathrm{h}$ post-infection), the nuclear of infected cell split into condensed fragments completely.

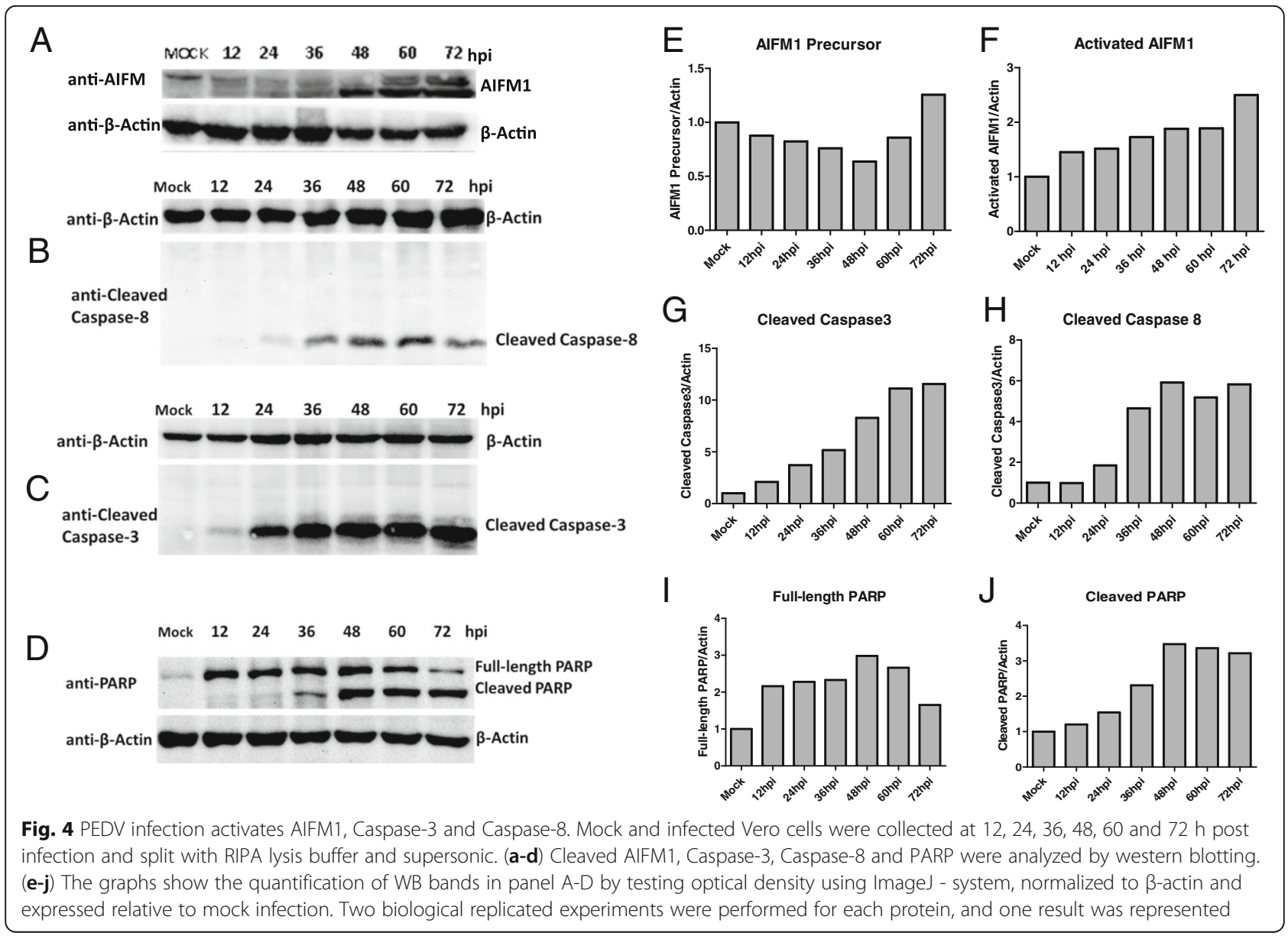


PEDV infection activates AIFM1, Caspase-8 and Caspase-3 To investigate the molecular mechanism of PEDV-induced apoptosis in host cells, we detected the timecourse activation status of AIFM1, caspase-3, caspase-8 in PEDV-infected Vero cells. The results showed that AIFM1 was cleaved upon PEDV infection. The amount of truncated AIFM1 was increased gradually (Fig. 4a). The cleaved caspase- 8 was also detected at $24 \mathrm{~h}$ post infection (B), while the cleavage of caspase- 9 was not observed in the whole course. Consistent with AIFM1 and caspase- 8 activation, the downstream effector caspase-3 (C) and substrate PARP (D) were also cleaved in response to PEDV infection. This together suggested PEDV infection not only activates AIFM1 but also induces the extrinsic apoptotic pathway activation. The relative changes of Western blot band intensity of AIFM1 precursor and activated AIFM1, as well as the cleaved caspases-3, -8 and PARP to $\beta$-actin were shown in Fig. $4 \mathrm{e}-\mathrm{j}$.

The effect of PEDV infection on caspase-3, -8 and -9 activations was further confirmed by using caspase activity assay kits at $48 \mathrm{~h}$ post infection. The relative fold changes $\left(\mathrm{OD}_{405} \mathrm{~nm}\right)$ of caspase-3, caspase- 8 and caspase- 9 in the PEDV infected cell lysis were shown in Fig. 5. The results showed that PEDV infection results in obviously Caspase3 activation and caspase- 8 activation, but no activation of caspase-9. The results are also consistent with above western-blot results.

\section{SM98 strain expression plasmids construction and fusion protein fluorescent identification}

To investigate the functional gene that is responsible for PEDV-induced apoptosis. We cloned all 16 nonstructural genes (Nsp1-16) and 6 structural genes including M, N, E, ORF3, S1 and S2 from PEDV SM98 strain and inserted them into the pEGFP-N1 vector.
These recombinant plasmids were identified by sequencing and fluorescence microscopy. The bright green fluorescence was observed from these recombinant plasmids-transfected cells, although the expression efficiency of each recombinant plasmid is different due to individual gene specificity (Additional file 2: Figure S1 and Additional file 3: Figure S2). This indicated that these PEDV structural and non-structural genes are correctly constructed and fused well with the EGFP gene.

\section{S1 protein is a critical inducer of apoptosis in host cells} Next, we detected the effect of the individual gene on cell apoptosis. The empty pEGFP-N1 vector and the recombinant plasmids infusion with the nonstructural proteins (Nsp1-16) and structural proteins (M, N, E, S1, S2 and ORF3) were transfected into 90\% confluent Vero E6 monolayer cells separately. $48 \mathrm{~h}$ after transfection, cells were stained with AnnexinV-PE and 7-AAD and detected by fluorescence-activated cells sorter (FACS), GFP-positive cells were gated for apoptosis analysis. Among all the structural proteins of PEDV SM98 strain, $\mathrm{S} 1$ protein has the predominant capability to induce apoptosis while nuclear protein $\mathrm{N}$, spike protein $\mathrm{S} 2$ and ORF3 have no effect on apoptosis induction. Membrane protein (M) and Nonstructural protein Nsp1, Nsp3, Nsp5, Nsp6 and Nsp7 also induce cell apoptosis but to variable less extents, as shown in Fig. 6a. Percentages of Annexin-V-PE positive cells from gated cells are shown with graphs in Fig. 6b. Percentages of Annexin-V-PE and 7AAD double positive cells from gated cells are shown with graphs in Fig. 6c. This suggests that S1 protein probably is the most critical protein-mediated PEDV-induced apoptosis.
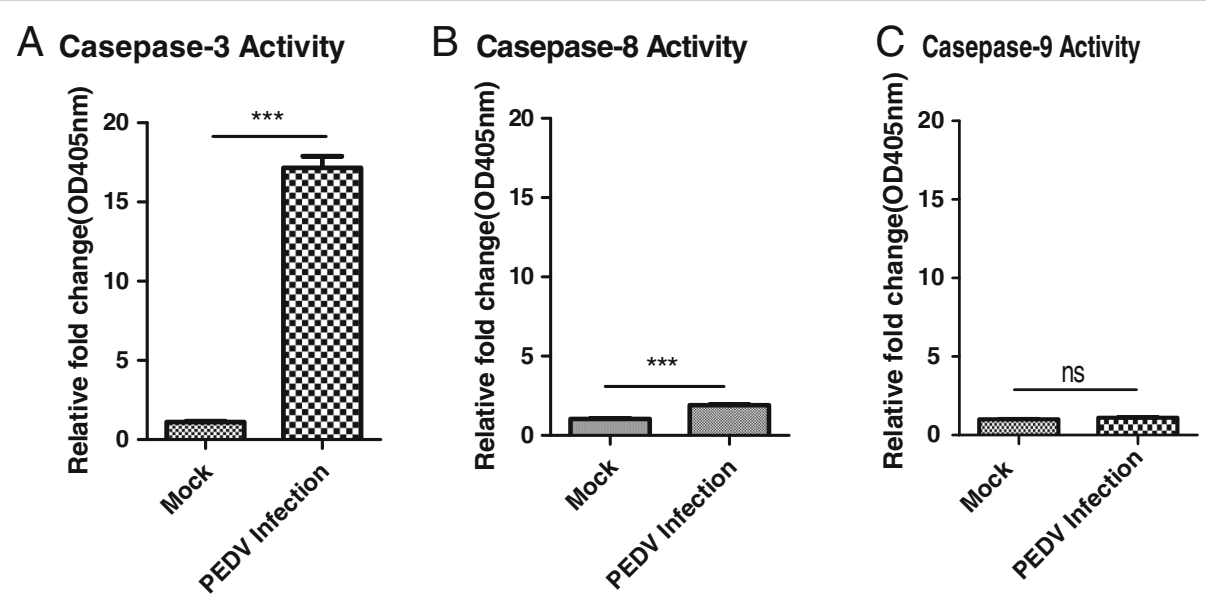

Fig. 5 PEDV infection results in the activation of Caspase-3 and Caspase-8,but not Caspase-9. The activation of Caspase-3, Caspase-8 and Caspase9 were detected by the Caspase activity assay kits. The relative fold change (OD405nm) of Caspase- $3,-8$ and -9 activities are shown with graphs. Data are represented as mean $+/-\mathrm{SD}, n=3$. (*** $^{*} p<0.001 ; \mathrm{ns}$, no significant) 


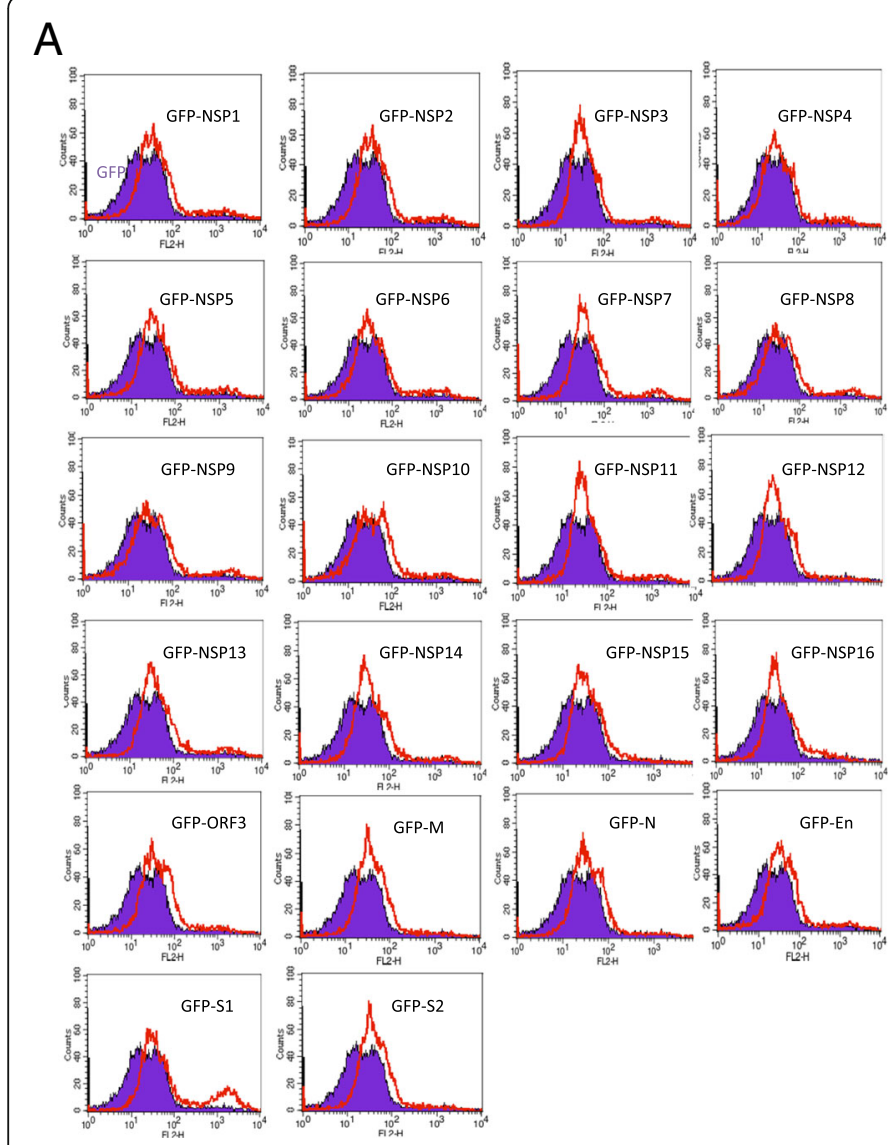

B
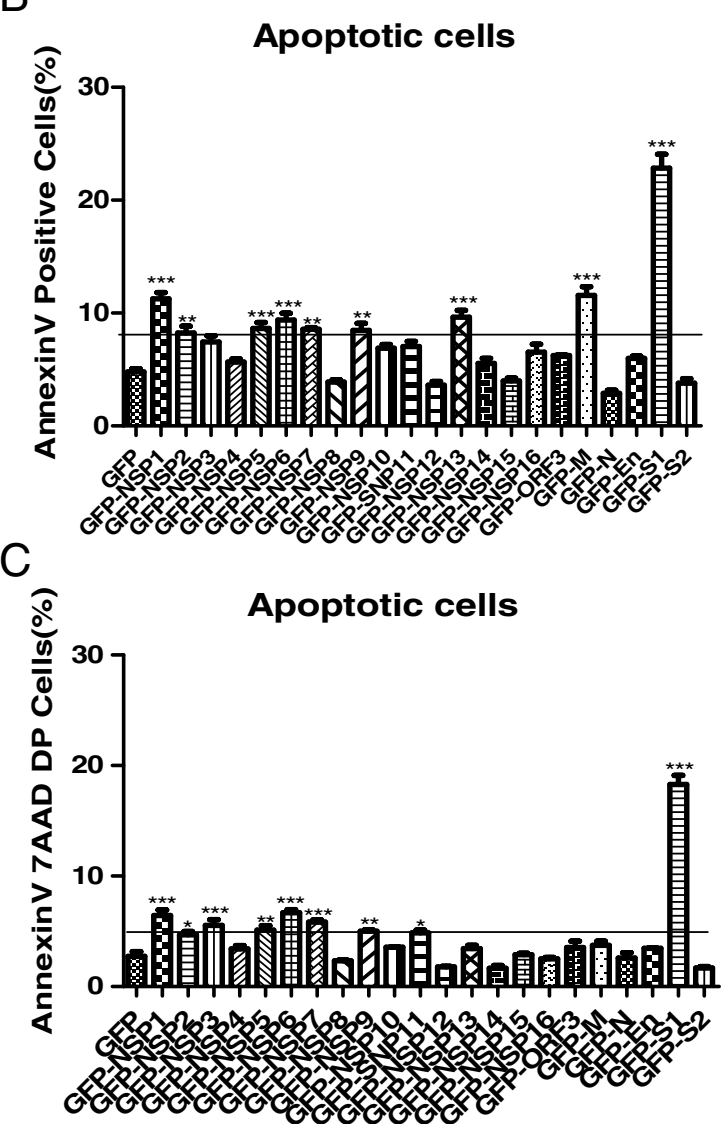

Fig. 6 PEDV S1 Protein is the Critical inducer of apoptosis among all the Structural and Nonstructural Proteins. pEGFP-N1 and all the recombinant plasmids were transfected into confluent Vero E6 monolayer cells respectively. $48 \mathrm{~h}$ later, the cells were harvested and analyzed by Fluorescenceactivated cells sorter (FACS), GFP-positive cells were gated for apoptosis analysis. (a)The results showed that PEDV S1 Protein is the Critical inducer of Apoptosis in host Cells. (b) Percentages of annexin-V-PE positive cells from gated cells. (c) Percentages of annexin-V-PE and 7AAD double positive cells from gated cells. Results are representative of three independent experiments. Results are representative of three independent experiments. Data are represented as mean $+/-\mathrm{SD}, \mathrm{n}=3 .\left(^{*}, p<0.05 ;^{* *}, p<0.01 ;{ }^{* *}, p<0.001\right)$

The S1 proteins in new-emerging and cell adapted PEDV strains also induce cell apoptosis

Next, we asked whether the S1 protein of other different PEDV strains could also induce apoptosis in different host cells. The S1 genes of CV777, a cell-adapted PEDV strain, and BJ2011, a new emerging wild-type PEDV isolated from Beijing in 2011 (The relationship of these PEDV $S$ proteins has been analyzed and reported previously in our laboratory) [20], were constructed in fusion with EGFP in pEGFP-N1 vector. Surprisingly, transfection S1 gene of CV777 and BJ2011 effectively induce monolayer Vero-E6 cells apoptosis. The similar result was also observed in transfected BHK-21 cells (Fig. 7a). Collectively, this result suggested that $\mathrm{S} 1$ protein is the determinant protein in PEDV infection-induced cell apoptosis, and this function is less dependent on virus biological or genetic differences. Percentages of apoptotic cells are shown with graphs in Fig. 7b and c respectively. This suggested that $\mathrm{S} 1$ protein probably is the most critical protein-mediated PEDV-induced apoptosis.

The S1 protein from various coronavirus resources induce apoptosis in Vero-E6 cells

Finally, we explored the pro-apoptotic function of the S1 protein in other coronavirus such as transmissible gastroenteritis virus (TGEV), canine coronavirus (CCoV), avian infectious bronchitis virus (IBV), severe acute respiratory syndrome coronavirus (SARS-CoV) and middle east respiratory syndrome coronavirus (MERS-CoV). The S1 gene from these coronaviruses was successfully cloned or synthesized, and infused with EGFP in pEGFP-N1 vector (Fig. 8a). The apoptosis potential of this $S 1$ protein was analyzed by FACS. As shown in Fig. 8b, S1 protein from all these coronaviruses could induce Vero-E6 cells apoptosis (Fig. 8c). Altogether, these data suggested that S1 protein have a 
A
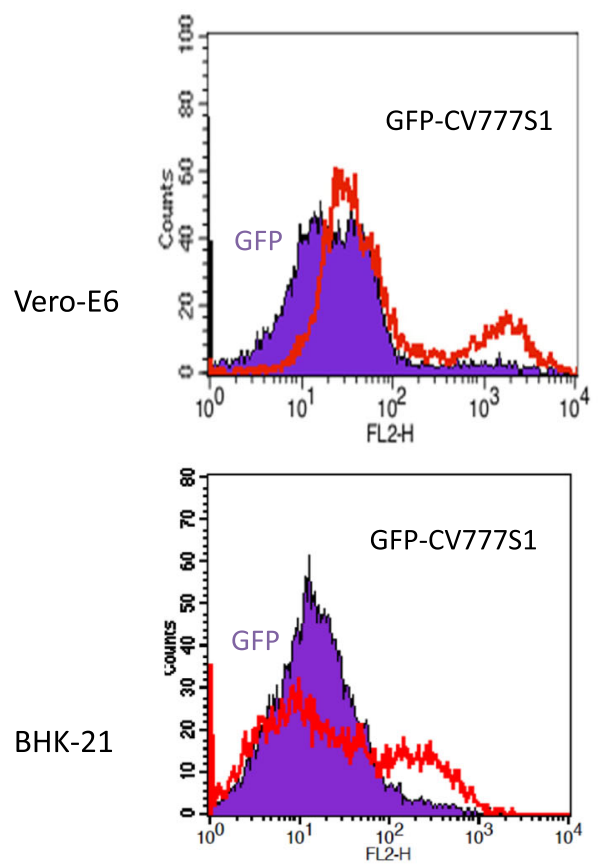
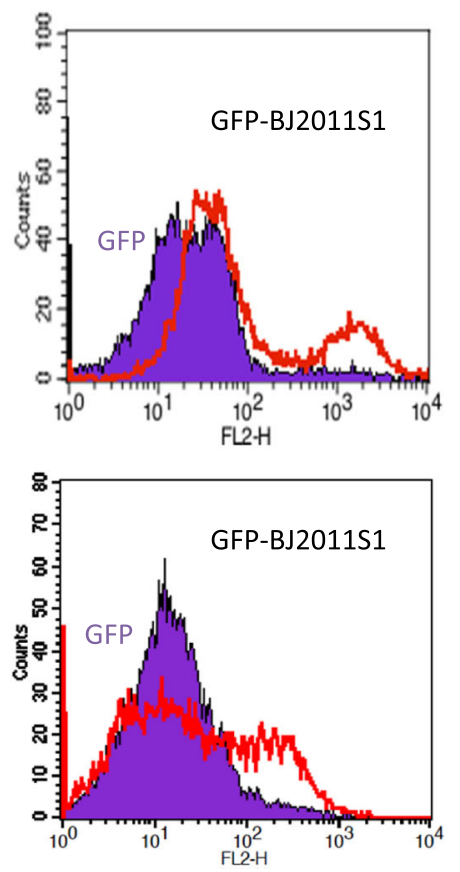

B
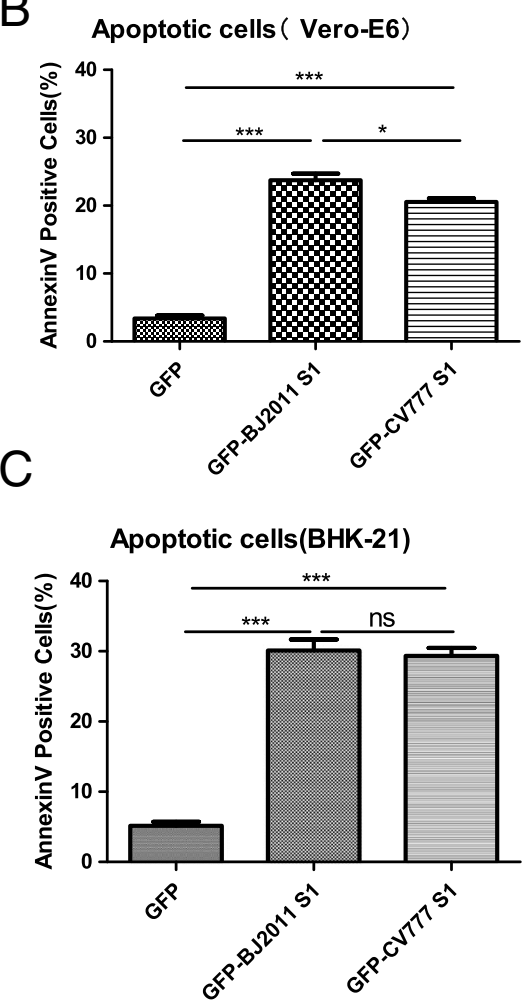

Fig. 7 PEDV CV777 and new-emerging strain BJ2011 S1 protein Could also induce apoptosis in VERO-E6 even in BHK-21 cells. pEGFP-N1 and the recombinant plasmids expressing CV777 or BJ2011 S1 protein were transfected into confluent Vero-E6 or BHK-21 monolayer cells respectively. $48 \mathrm{~h}$ later, the cells were harvested and analyzed by Fluorescence-activated cells sorter (FACS), GFP-positive cells were gated for apoptosis analysis. (a) PEDV CV777 and new-emerging strain BJ2011 S1 protein Could also induce apoptosis in Vero-E6 even in BHK-21 cells. (b) Percentages of annexin-V-PE positive Vero-E6 Cells from gated cells are shown with graphs. Results are representative of three independent experiments. (c) Percentages of annexin-V-PE positive BHK-21 Cells from gated cells are shown with graphs. Results are representative of three independent experiments. ( ${ }^{*}, p<0.05 ;{ }^{* *}, p<0.01 ;{ }^{* *}, p<0.001$; ns, no significant)

general function inducing cell apoptosis in the coronaviruses, targeting $\mathrm{S} 1$ protein probably is a promising strategy to inhibit coronavirus infection and thus decreases the threat to animal industry and human health.

\section{Discussion}

In this study, we observed that PEDV SM98 and CV777 strains induce apoptosis in Vero, Vero-E6 and Marc-145 cells with distinct cytopathic effects (CPEs) including roundup, cell fusion, vacuolation, syncytium formation etc. Membrane blebbing and the translocation of phosphatidi lserine to the cell surface, as well as nuclear concentration and fragmentation, were all observed in PEDV infected cells.

Mechanically, PEDV SM98 and CV777 virus infection induced a significant cleavage on AIFM1 suggested an involvement of mitochondrial-mediated apoptosis pathway, which is consistent with previous observation [19]. Besides this pathway, we found the extrinsic apoptotic pathway was also activated by SM98 and CV777 infection as caspase-8, caspase 3 and PARP were all cleaved in response to PEDV virus infection. This study enriched the knowledge of the PEDV infection induced apoptosis through a complicated pattern, apart from the mitochondrial-mediated apoptosis pathway, the extrinsic apoptotic pathway was also activated, no matter whether or not it is original. Consistently, many viruses from coronavirus family induced apoptosis with caspase activation: such as TGEV could cause infected ST cells apoptosis in a caspase-dependent way, accompanied by DNA cleavage and mitochondrial transmembrane potential change [21]; IBV could cause a caspase-dependent apoptosis, as characterized by chromosomal condensation, DNA fragmentation, caspase-3 activation, and poly (ADP-ribose) polymerase degradation [22]; $\mathrm{CCoV}$, is responsible for enteric disease (diarrhea, vomiting, dehydration, loss of appetite and occasional death) in young puppies [23, 24], could cause infected cells apoptosis with activation caspase-3 [23]; SARS-CoV infection could results in the downregulation of $\mathrm{Bcl}-2$, the activation of caspase-3, as well as the upregulation of Bax, suggesting the involvement of the caspase family and the activation of the mitochondrial signaling pathway [25]. MERS-CoV could 

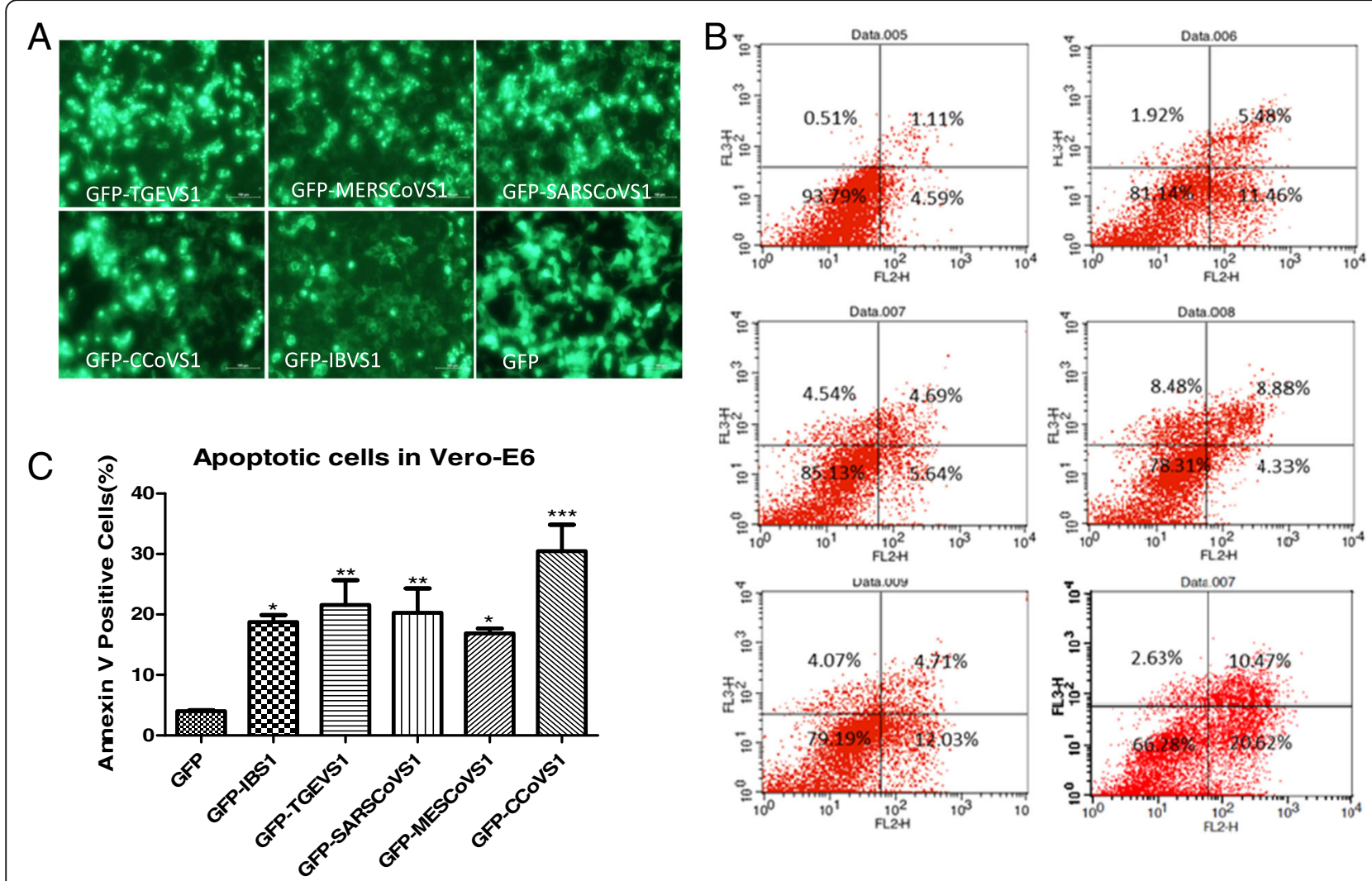

Fig. 8 TGEV, IBV, COCoV, SARS and MERS CoV Spike Protein S1 Could also Induce Apoptosis in Vero-E6 cells. TGEV, IBV, CCoV, SARS and MERS S1 genes were cloned or synthesized in fusion with EGFP gene and were transfected into VERO-E6, $48 \mathrm{~h}$ later, the cells were harvested and analyzed by Fluorescence-activated cells sorter (FACS), GFP-positive cells were gated for apoptosis analysis. (a) TGEV, IBV, CCoV, SARS and MERS S1 genes were correctly constructed in fusion with EGFP. The empty vector pEGFP-N1 and the recombinant plasmids were all transfected into confluent BHK-21 monolayer cells separately. $24 \mathrm{~h}$ later, bright green fluorescence was observed from the all the recombinant plasmids transfected cells. (b) The results showed that TGEV, IBV, CCOV, SARS and MERS S1 Protein Could Induce Apoptosis in Vero-E6 cells. (c)Percentages of annexin-V-PE positive cells from gated cells are shown with graphs. Results are representative of three independent experiments. $\left({ }^{*}, p<0.05 ;{ }^{* *}, p<0.01\right.$; ***, $p<0.001)$

efficiently infect human primary $\mathrm{T}$ lymphocytes and activates the extrinsic and intrinsic apoptosis pathway [26]. However, the exact mechanism especially the critical viral inducer of PEDV has not yet illustrated.

To date, PEDV has evolved into two distinct clades, the classical strains and high-virulent field strains [14]. Antigenic variations of PEDV spike protein (S) between these two groups were thought to contribute to the severity of recent outbreaks $[27,28]$, as the $\mathrm{S}$ protein plays a pivotal role in cell adsorption, membrane fusion, and induction of neutralizing antibodies $[29,30]$. Here we demonstrate that S1 protein indeed is a predominant apoptosis inducer (while S2 is not) among all the structural and nonstructural proteins of PEDV, although $M$ and Nsp1, 2, 5, 6, 7, 9 and 13 also induce cell apoptosis but to a less extent. Furthermore, the S1 protein of circulating variant strain PEDV BJ2011 also induce significant apoptosis in host cells, and the ability to induce apoptosis in Vero-E6 is greater than that of the cell adapted strain, such as CV777. This suggested S1 protein is a critical protein mediated PEDV-induced cell apoptosis and contributes partly to its virulence, which is consistent with our laboratory's recent report [31]. Among all the identified proteins capable inducing apoptosis in host cells, the $M$ protein is the most abundant component of the viral envelope and plays a central role in virus morphogenesis and assembly via its interactions with other viral proteins [32]. The pro-apoptotic role of the SARS-CoV M protein has been revealed by disrupting the interaction of PDK1 with $\mathrm{PKB} /$ Akt $[33,34]$. This suggests that PEDV M protein may also activate apoptosis through this pathway. 3C-like protease (3CLpro) has been demonstrated could cause cell growth arrest and apoptosis in SARS-CoV 3CLpro-expressing human promonocyte cells [35]. Similarly, the pro-apoptotic function of PEDV Nsp5 i.e. 3C-like protease also has observed in this study. Coronavirus Nsp1 is the first N-terminal cleavage product of the $\mathrm{ppla}$ and $\mathrm{ppla} / \mathrm{b}$ polyproteins [36]. Coronavirus Nsp1 regulates host cell and virus gene expression [37] that functions as a potent IFN antagonist. PEDV Nsp1 has been demonstrated could block 
the nuclear translocation of IRF1 and reduce the number of peroxisomes to suppress IRF1-mediated type III IFNs activities [38]. Here we identified a novel function of PEDV Nsp1 as an inducer of apoptosis.

Coronaviruses can infect a wide range of mammals and birds, but exhibit a marked tropism for epithelial cells of the respiratory and enteric tracts, as well as for macrophages [16, 39], and most of them can induce apoptosis in infected host cells. Although a number of cellular mechanisms and gene products (such as the SARS-CoV M, N protein[34, 40] and 3C-like protease (3CLpro) [35]) capable of inducing apoptosis have been revealed, the S1 protein of these coronaviruses have not yet well-evaluated. Although the amino acid sequence of these $\mathrm{S}$ proteins are divergent greatly (Additional file 1: Table S1), here we demonstrated that $\mathrm{S} 1$ proteins of all these coronaviruses tested in this study were able to induce apoptosis in transfected Vero-E6 cells. Given the fact that a number of coronaviruses gene products could induce apoptosis in host cells, it is proposed that the spike protein S1 may function as a cofactor that could enhance or stimulate extrinsic apoptotic pathway in these coronavirus induced apoptosis.

\section{Conclusion}

PEDV Spike protein S1 is one of the most critical functional proteins that contributes to cell apoptosis,.Expression of S1 proteins of the coronavirus tested in this study could all induce cell apoptosis suggesting S1 maybe is an effective inducer in Coronavirus-induced apoptosis and targeting S1 protein expression probably is a promising strategy to inhibit coronavirus infection and thus mediated apoptosis on host cells.

\section{Additional files}

Additional file 1: Table S1. Primers for genes cloning and amplication. (DOCX $19 \mathrm{~kb}$ )

Additional file 2: Figure. S1. Fluorescent identification of recombinant plasmids. (PPTX $2490 \mathrm{~kb}$ )

Additional file 3: Figure S2. Homology and phylogenetic tree analysis of $\mathrm{S}$ proteins utilized in the study. (PPTX $151 \mathrm{~kb}$ )

\section{Abbreviations \\ AlFM1: Apoptosis-inducing factor mitochondria-associated 1; CCoV: Canine coronavirus; FACS: Fluorescence-activated cells sorter; IBV: Infectious bronchitis virus; mAb: Monoclonal antibody; MERS: Middle East respiratory syndrome; Nsp: Non-structural protein; ORF: Open reading frame; PARP: Poly ADP-ribose polymerase; PBS: Phosphate buffered saline; PEDV: Porcine reproductive and respiratory syndrome virus; RT: Room temperature; SARS: Severe acute respiratory syndrome; TGEV: Transmissible gastroenteritis virus}

\section{Acknowledgements}

Not applicable.

\section{Funding}

The study was supported by the earmarked fund for China Agriculture Research System from the Ministry of Agriculture of China (grant CARS-36)
Availability of data and materials

Data and materials related to this work are available upon request

\section{Authors' contributions}

$X \mathrm{X}$ and $\mathrm{H}-\mathrm{CY}$ designed the study, $\mathrm{Y}-\mathrm{FC}$ performed most of the experiments and drafted the manuscript. ZB-Z, J-L and $Y-Y G$ performed some of the experiments. LZ and X-NG contributed to the study design. JH supervised the work and $X G$ revised the manuscript. All authors read and approved the final manuscript.

Ethics approval and consent to participate

Not applicable

\section{Consent for publication}

Not applicable.

\section{Competing interests}

The authors declare that they have no conflict of interest

\section{Publisher's Note}

Springer Nature remains neutral with regard to jurisdictional claims in published maps and institutional affiliations.

\section{Author details}

${ }^{1}$ Key Laboratory of Animal Epidemiology of the Ministry of Agriculture, College of Veterinary Medicine and State Key Laboratory of Agrobiotechnology, China Agricultural University, No.2 Yuanmingyuan West Road, Haidian Distract, Beijing 100193, People's Republic of China. ${ }^{2}$ Animal Medicine Research Center of DBN Group, South Crossroad of Xiangrui Street and Huatuo Road DBN Daxing Science Park, Daxing Distract, Beijing 102600, People's Republic of China.

Received: 8 March 2018 Accepted: 10 October 2018 Published online: 07 November 2018

\section{Referecnces}

1. Chasey D, Cartwright SF. Virus-like particles associated with porcine epidemic diarrhoea. Research in veterinary science. 1978;25(2):255-6.

2. Pensaert MB, de Bouck P. A new coronavirus-like particle associated with diarrhea in swine. Archives of virology. 1978;58(3):243-247.

3. Kusanagi K, Kuwahara H, Katoh T, Nunoya T, Ishikawa Y, Samejima T, et al. Isolation and serial propagation of porcine epidemic diarrhea virus in cell cultures and partial characterization of the isolate. The Journal of veterinary medical science / the Japanese Society of Veterinary Science. 1992;54(2):313-8.

4. Lee HM, Lee BJ, Tae JH, Kweon CH, Lee YS, Park JH. Detection of porcine epidemic diarrhea virus by immunohistochemistry with recombinant antibody produced in phages. The Journal of veterinary medical science / the Japanese Society of Veterinary Science. 2000;62(3):333-7.

5. Chen J, Wang C, Shi H, Qiu HJ, Liu S, Shi D, et al. Complete genome sequence of a Chinese virulent porcine epidemic diarrhea virus strain. Journal of virology. 2011;85(21):11538-9.

6. Bi J, Zeng S, Xiao S, Chen H, Fang L. Complete genome sequence of porcine epidemic diarrhea virus strain AJ1102 isolated from a suckling piglet with acute diarrhea in China. Journal of virology. 2012;86(19):10910-1.

7. Chen F, Pan Y, Zhang X, Tian X, Wang D, Zhou Q, et al. Complete genome sequence of a variant porcine epidemic diarrhea virus strain isolated in China. Journal of virology. 2012;86(22):12448.

8. Li W, Li H, Liu Y, Pan Y, Deng F, Song Y, et al. New variants of porcine epidemic diarrhea virus, China, 2011. Emerging infectious diseases. 2012;18(8):1350-3.

9. Davies PR. The dilemma of rare events: Porcine epidemic diarrhea virus in North America. Preventive veterinary medicine. 2015.

10. Hoang H, Killian ML, Madson DM, Arruda PH, Sun D, Schwartz KJ, et al. FullLength Genome Sequence of a Plaque-Cloned Virulent Porcine Epidemic Diarrhea Virus Isolate (USA/lowa/18984/2013) from a Midwestern U.S. Swine Herd. Genome Announc. 2013;1(6).

11. Huang YW, Dickerman AW, Pineyro P, Li L, Fang L, Kiehne R, et al. Origin, evolution, and genotyping of emergent porcine epidemic diarrhea virus strains in the United States. mBio. 2013;4(5):e00737-13.

12. Stevenson GW, Hoang H, Schwartz KJ, Burrough ER, Sun D, Madson D, et al Emergence of Porcine epidemic diarrhea virus in the United States: clinical 
signs, lesions, and viral genomic sequences. J Vet Diagn Invest: official publication of the American Association of Veterinary Laboratory Diagnosticians, Inc. 2013;25(5):649-654.

13. Wang $L$, Byrum B, Zhang Y. New variant of porcine epidemic diarrhea virus, United States, 2014. Emerging infectious diseases. 2014;20(5):917-9.

14. Jaru-Ampornpan P, Jengarn J, Wanitchang A, Jongkaewwattana A. Porcine Epidemic Diarrhea Virus 3C-Like Protease-Mediated Nucleocapsid Processing: Possible Link to Viral Cell Culture Adaptability. Journal of virology. 2017;91(2).

15. Kocherhans R, Bridgen A, Ackermann M, Tobler K. Completion of the porcine epidemic diarrhoea coronavirus (PEDV) genome sequence. Virus genes. 2001;23(2):137-44

16. Lee C. Porcine epidemic diarrhea virus: An emerging and re-emerging epizootic swine virus. Virology journal. 2015;12(1):193.

17. Bosch BJ, van der Zee R, de Haan CA, Rottier PJ. The coronavirus spike protein is a class I virus fusion protein: structural and functional characterization of the fusion core complex. Journal of virology. 2003; 77(16):8801-8811.

18. Peng G, Sun D, Rajashankar KR, Qian Z, Holmes KV, Li F. Crystal structure of mouse coronavirus receptor-binding domain complexed with its murine receptor. Proceedings of the National Academy of Sciences of the United States of America. 2011;108(26):10696-701.

19. Kim Y, Lee C. Porcine epidemic diarrhea virus induces caspase-independent apoptosis through activation of mitochondrial apoptosis-inducing factor. Virology. 2014;460-461:180-93.

20. Gao Y, Kou Q, Ge X, Zhou L, Guo X, Yang H. Phylogenetic analysis of porcine epidemic diarrhea virus field strains prevailing recently in China. Archives of virology. 2013;158(3):711-5.

21. Eleouet JF, Slee EA, Saurini F, Castagne N, Poncet D, Garrido C, et al. The viral nucleocapsid protein of transmissible gastroenteritis coronavirus (TGEV) is cleaved by caspase- 6 and -7 during TGEV-induced apoptosis. Journal of virology. 2000;74(9):3975-83.

22. Liu C, Xu HY, Liu DX. Induction of caspase-dependent apoptosis in cultured cells by the avian coronavirus infectious bronchitis virus. Journal of virology. 2001;75(14):6402-9.

23. Ruggieri A, Di Trani L, Gatto I, Franco M, Vignolo E, Bedini B, et al. Canine coronavirus induces apoptosis in cultured cells. Veterinary microbiology. 2007;121(1-2):64-72

24. De Martino L, Marfe G, Longo M, Fiorito F, Montagnaro S, lovane V, et al. Bid cleavage, cytochrome $\mathrm{c}$ release and caspase activation in canine coronavirusinduced apoptosis. Veterinary microbiology. 2010;141(1-2):36-45.

25. Ren L, Yang R, Guo L, Qu J, Wang J, Hung T. Apoptosis induced by the SARS-associated coronavirus in Vero cells is replication-dependent and involves caspase. DNA and cell biology. 2005;24(8):496-502.

26. Chu H, Zhou J, Wong BH, Li C, Chan JF, Cheng ZS, et al. Middle East Respiratory Syndrome Coronavirus Efficiently Infects Human Primary $T$ Lymphocytes and Activates the Extrinsic and Intrinsic Apoptosis Pathways. In: The Journal of infectious diseases; 2015.

27. Lin CM, Annamalai T, Liu X, Gao X, Lu Z, El-Tholoth M, et al. Experimental infection of a US spike-insertion deletion porcine epidemic diarrhea virus in conventional nursing piglets and cross-protection to the original US PEDV infection. Veterinary research. 2015;46(1):134.

28. Lin CM, Gao X, Oka T, Vlasova AN, Esseili MA, Wang Q, et al. Antigenic relationships among porcine epidemic diarrhea virus and transmissible gastroenteritis virus strains. Journal of virology. 2015;89(6):3332-42.

29. Park SJ, Moon HJ, Yang JS, Lee CS, Song DS, Kang BK, et al. Sequence analysis of the partial spike glycoprotein gene of porcine epidemic diarrhea viruses isolated in Korea. Virus genes. 2007;35(2):321-32.

30. Wang J, Zhao P, Guo L, Liu Y, Du Y, Ren S, et al. Porcine epidemic diarrhea virus variants with high pathogenicity, China. Emerging infectious diseases. 2013;19(12):2048-9.

31. Wang D, Ge X, Chen D, Li J, Cai Y, Deng J, et al. The S Gene Is Necessary but Not Sufficient for the Virulence of Porcine Epidemic Diarrhea Virus Novel Variant Strain BJ2011C. Journal of virology. 2018;92:13.

32. Zhang Z, Chen J, Shi H, Chen X, Shi D, Feng L, et al. Identification of a conserved linear B-cell epitope in the M protein of porcine epidemic diarrhea virus. Virology journal. 2012;9:225.

33. Chan CM, Ma CW, Chan WY, Chan HY. The SARS-Coronavirus Membrane protein induces apoptosis through modulating the Akt survival pathway. Archives of biochemistry and biophysics. 2007:459(2):197-207.
34. Tsoi H, Li L, Chen ZS, Lau KF, Tsui SK, Chan HY. The SARS-coronavirus membrane protein induces apoptosis via interfering with PDK1-PKB/Akt signalling. The Biochemical journal. 2014;464(3):439-47.

35. Lin CW, Lin KH, Hsieh TH, Shiu SY, Li JY. Severe acute respiratory syndrome coronavirus 3C-like protease-induced apoptosis. FEMS immunology and medical microbiology. 2006;46(3):375-80.

36. Ziebuhr J. The coronavirus replicase. Current topics in microbiology and immunology. 2005;287:57-94.

37. Kamitani W, Narayanan K, Huang C, Lokugamage K, Ikegami T, Ito N, et al. Severe acute respiratory syndrome coronavirus nsp1 protein suppresses host gene expression by promoting host mRNA degradation. Proceedings of the National Academy of Sciences of the United States of America. 2006; 103(34):12885-90.

38. Zhang Q, Ke H, Blikslager A, Fujita T, Yoo D. Type III Interferon Restriction by Porcine Epidemic Diarrhea Virus and the Role of Viral Protein nsp1 in IRF1 Signaling. Journal of virology. 2018;92(4).

39. Reguera J, Mudgal G, Santiago C, Casasnovas JM. A structural view of coronavirus-receptor interactions. Virus research. 2014;194:3-15.

40. Zhao G, Shi SQ, Yang Y, Peng JP. M and N proteins of SARS coronavirus induce apoptosis in HPF cells. Cell biology and toxicology. 2006;22(5):313-22.

\section{Ready to submit your research? Choose BMC and benefit from:}

- fast, convenient online submission

- thorough peer review by experienced researchers in your field

- rapid publication on acceptance

- support for research data, including large and complex data types

- gold Open Access which fosters wider collaboration and increased citations

- maximum visibility for your research: over $100 \mathrm{M}$ website views per year

At BMC, research is always in progress.

Learn more biomedcentral.com/submissions 